\title{
On the Three-Taxon Approach to Parsimony Analysis
}

\author{
Jan De Laet and Erik Smets \\ Laboratorium voor Systematiek, Instituut voor Plantkunde, K.U.Leuven, Kard. M ercierlaan 92, B-3001 Heverlee, \\ Belgium
}

Received for publication 22 September 1998

The following three basic defects for which three-taxon analysis has been rejected as a method for biological systematics are reviewed: (1) character evolution is a priori assumed to be irreversible; (2) basic statements that are not logically independent are treated as if they are; (3) three-taxon statements that are considered as independent support for a given tree may be mutually exclusive on that tree. It is argued that these criticisms only relate to the particular way the three-taxon approach was originally implemented. Four-taxon analysis, an alternative implementation that circumvents these problems, is derived. Four-taxon analysis is identical to standard parsimony analysis except for an unnatural restriction on the maximum amount of homoplasy that may be concentrated in a single character state. This restriction follows directly from the basic tenet of the three-taxon approach, that character state distributions should be decomposed into basic statements that are, in themselves, still informative with respect to relationships. A reconsideration of what constitutes an elementary relevant statement in systematics leads to a reformulation of standard parsimony as two-taxon analysis and to a rejection of four-taxon analysis as a method for biological systematics.

(c) 1998 The Willi Hennig Society

\section{INTRODUCTION}

Nelson and Platnick (1991) introduced three-taxon analysis as a novel approach to parsimony analysis in systematics. A similar approach, three-area analysis, has been proposed for use in biogeography (Nelson and Ladiges, 1991a, 1991b). The names of these methods refer to the fact that each statement about relationships between more than three items (taxa in systematics, areas in biogeography) is decomposed into a series of basic statements, each of which involves only three items. Such a basic statement states which two of the three items are thought to be related more closely to each other than either is related to the third. Cladograms that accommodate the highest number of such basic statements are considered the best cladograms for the data. It was suggested (Nelson and Ladiges, 1991a, 1991b; Nelson and Platnick, 1991) that this approach might increase the precision of parsimony, i.e. its sensitivity to differences in the fit of data to alternative cladograms. Practical applications of three-taxon analysis, using a set of computer programs written by Nelson and Ladiges (1995), can be found in Nelson and Ladiges (1994), Patterson and Johnson (1995) and Udovicic et al. (1995). The use of three-item analysis in biogeography has been further explored by Ladiges et al. (1992), Morrone and Carpenter (1994) and Nelson and Ladiges (1996), amongst others. The theoretical justification for using three-item statements in systematics on the one hand and biogeography on the other may be different (Nelson, 1992); this paper is concerned with systematics.

Three-taxon analysis has been criticized from different points of view and for numerous reasons (Harvey, 1992; Kluge, 1993, 1994; Wilkinson, 1994; De

0748-3007/98/040401+ $10 \$ 30.00 / 0$ 
Laet and Smets, 1995; Farris et al., 1995; Deleporte, 1996). Superficially, the points of criticism seem to involve many different and unrelated aspects, but closer inspection reveals that all criticisms can be reduced to only three basic problems (De Laet, 1997). Three-taxon analysis is unsuited as a method for biological classification because: (1) it presupposes that character evolution is irreversible; (2) it treats basic statements that are not logically independent as if they are; (3) some of the basic statements considered as independent support for a given tree may be mutually exclusive on that tree. Mostly as a response to these criticisms, Nelson and Platnick (Nelson, 1992, 1993, 1996; Platnick, 1993; Platnick et al., 1996; see also Nelson and Ladiges, 1992, 1993, 1994) have further explained and clarified the three-item approach. However, as discussed below, none of the basic criticisms has been answered adequately. De Laet and Smets (1995) proposed four-taxon analysis as a solution to the first problem, but this modification of three-taxon analysis still has problems associated with dependence and mutual exclusiveness. In this paper we will more fully describe four-taxon analysis as proposed by De Laet and Smets (1995), and examine how it can be further refined. The resulting modification of four-taxon analysis will subsequently be used to evaluate the differences between standard parsimony and the three-taxon approach. We will first briefly discuss three-taxon analysis to provide a basis for further argument.

\section{THREE-TAXON ANALYSIS}

In the standard approach to parsimony analysis, character state distributions are typically given in the form of a matrix with the rows representing taxa and the columns characters. An example, showing the character state distributions of two binary characters over five taxa, is given in Fig. 1 (left). In standard parsimony analysis (Farris, 1983) these character state distributions are fitted in their entirety onto cladograms, so that the congruency between characters and cladograms is maximized. The two basic intuitions behind three-taxon analysis (Nelson and Platnick, 1991) seem to be: (1) the idea that the state distribution of a single character is a compound state- ment that might somehow be further decomposed into more basic statements; and (2) the idea that a parsimony analysis on the level of these basic statements might provide a better measure of congruence between data and cladograms. The nature of such basic statements then becomes a central question.

The smallest possible statement that is still informative about relative cladistic branching necessarily involves three taxa (e.g. 'taxa A and B are related more closely to each other than either is to $C^{\prime}$ ), which seems to be Nelson and Platnick's (1991) rationale for concentrating on three-taxon statements that homologize a feature that is only found in two out of three taxa (called the "smallest relevant observation about character diversity" by Platnick et al., 1996; see also Nelson, 1994: 128-129). Such a statement hypothesizes that, on the basis of the character involved, the two taxa with the homologized feature (i.e. the apomorphic character state) are more closely related to each other than either is to the third; thus they belong to a monophyletic group from which the third is excluded.

Following this line of reasoning, the first step of three-taxon analysis is the decomposition of the character state distribution of each character under study into the series of such statements implied by the distribution (Fig. 1). The decomposition is mostly called a "transformation", often with the negative connotation that the information content of the data is being distorted. This issue has caused a great deal of debate (e.g. Harvey, 1992; Kluge, 1993; Nelson, 1992, 1993; see also below for a discussion of exactly what constitutes the information content of systematic data). In making the decomposition, Nelson and Platnick (1991) implicitly make the assumption that for each character the apomorphic state has been determined a priori. This assumption is necessary because, in general, both the presence of a structure and its absence due to loss can be a homology (Farris, 1983: 20; note that there is no connection with naive grouping on the basis of absence of structures, or with grouping by plesiomorphy). For each character, the apomorphic state is by convention coded as 1 and the plesiomorphic state as 0 . A hypothetical outgroup taxon that is coded 0 for all characters is added to the data set. Because 0 is assumed to be plesiomorphic, only 0-1-1 three-taxon statements (i.e. a statement about one taxon having state 0 and two taxa having 
Standard approach

Characters

Taxa $a b$

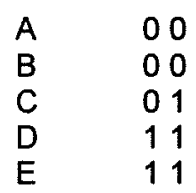

Three-taxon approach

$\begin{array}{cccc} & & a & b \\ \text { "Transformation" } & \text { O } & 000 & 000000 \\ \rightarrow & \text { A } & 0 ? ? & 000 ? ? ? \\ & \text { B } & ? 0 ? & ? ? ? 000 \\ \text { C } & ? ? 0 & 11 ? 11 ? \\ & \text { D } & 111 & 1 ? 11 ? 1 \\ & \text { E } & 111 & ? 11 ? 11\end{array}$

FIG. 1. The representation of the character state distribution of two characters, a and b, over five taxa, A-E. Left: representation using the standard approach. Right: representation using the three-taxon approach; each column represents one three-taxon statement; the character state distribution of character a implies three three-taxon statements, character b implies six three-taxon statements; an outgroup (O) is added to indicate that 0 represents the plesiomorphic state, "?" is used to indicate taxa that are not part of a particular statement.

state 1) have to be considered; 0-0-1 statements are not informative with respect to cladistic branching; they merely indicate that two out of three taxa have retained a plesiomorphic character state. It follows that a complete transformed representation of the character state distribution of a single character consists of the set of all possible 0-1-1 three-taxon statements that can be derived from the standard representation of the character. In this way the number of informative three-taxon statements implied by a character state distribution depends on the numbers of taxa that have the apomorphic state (no) and the plesiomorphic state (nz) for that character; $n z^{*}$ no*(no-1)/2. In a three-taxon matrix, each column represents a single three-taxon statement (Fig. 1). In each statement, the three taxa involved are indicated using their character state ( 0 or 1 ) and the remaining taxa are indicated by question marks. The question marks do not indicate any type of missing data (Platnick et al., 1991); they are only inserted to allow the use of computer programs for standard parsimony in the second step of three-taxon analysis.

In this second step, the matrix consisting of the three-taxon statements is subjected to standard parsimony analysis, using any of the standard programs available (e.g. Farris, 1988; Swofford, 1993; Goloboff, 1993). The all-zero hypothetical outgroup is included as a technical necessity to effectively force character state zero into the plesiomorphic role. An individual three-taxon statement either fits a cladogram or does not, therefore the resulting cladograms will be those that maximize the number of three-taxon statements that can be accommodated. Each of these accommodated statements is interpreted as a valid indicator of monophyly (Nelson and Platnick, 1991).

\section{AVOIDING A PRIORI IRREVERSIBILITY: FOUR-TAXON ANALYSIS}

In the standard approach to parsimony analysis, the possibility exists to construct most-parsimonious trees under the strong assumption that all character evolution is in a forward direction: once a derived character state has evolved it will never revert to the plesiomorphic state. Consequently, all homoplasy is explained in terms of convergence, and reversals are not allowed. With the assumptions that character polarity and, for multistate characters, character state order can be determined prior to the parsimony analysis, this has been called Camin-Sokal parsimony (e.g. Swofford et al., 1996; cf. Camin and Sokal, 1965: 312). At first sight, it may appear that only a priori polarization is involved in three-taxon analysis, however, the decision not to include 0-0-1 three-taxon statements in the three-taxon matrix also implies the stronger assumption of irreversibility. Indeed, 0-0-1 statements are omitted from the matrix because they are assumed to be uninformative, but this is only so if it is assumed that state 1 never can be plesiomorphic with respect to a reverted state 0 . If three-taxon analysis is indeed an alternative to standard parsimony analysis, it is only so under the very restrictive assumptions of Camin-Sokal parsimony (De Laet and Smets, 1995; see also Kluge, 1993: 251; Deleporte, 1996).

Standard Wagner parsimony (Kluge and Farris, 1969) and standard Fitch parsimony (Fitch, 1971) do not make the a priori assumption of irreversibility of character evolution. Under these conditions, it is no longer possible to determine a priori whether a given 
$0-1-1$ or $0-0-1$ three-taxon statement will be informative or not. Nelson and Platnick (1991: 362-363; see also Platnick, 1993: 268) considered the possibility that reversals could be problematic in three-taxon analysis, but by providing a hypothetical data set they showed, by example, that three-taxon analysis can identify clades supported only by reversals ${ }^{1}$. However, Kluge $(1993,1994)$ correctly pointed out that such hypothetical examples do not solve the basic problem: a three-taxon matrix is constructed in such a way that congruence can no longer be used to test putative symplesiomorphies as evolutionary reversals.

Platnick (1993: 268) argued that there is no real problem; in principle any individual character polarity could be altered in any possible combination of polarities of the other characters, and these alternative a priori polarities could be compared to achieve maximum congruence. However, rather than solving the problem, this suggestion merely reverses it for the individual characters: either one or the other state is assumed, a priori, to be plesiomorphic throughout the complete tree. For the same reason, it would not help to substitute the hypothetical outgroup for a real one.

If the basic intuition of three-taxon analysis is that character state distributions should be broken down into the smallest possible statements that are still informative with respect to cladistic relationships, a generalization that does not assume irreversibility or a priori polarization suggests itself. Consider a 0-0-1-1 four-taxon statement. Such a statement is always informative with respect to cladistic branching. Indeed, independent of the plesiomorphic state, a 0-0-1-1 four-taxon statement will either be accommodated on a particular tree (only one step required) or not (two steps required). The other possible types of four-taxon statements (0-0-0-0, 0-0-0-1, 0-1-1-1, and 1-1-1-1) are all uninformative because they require the same number of steps on any tree (no steps for $0-0-0-0$ and 1-1-1-1; one step for 0-1-1-1 and 0-0-0-1).

To denote a particular four-taxon statement, all taxa that have the same state will be put between

\footnotetext{
${ }^{1}$ Note that no such clade is identified when the data set is analysed as it is presented (Farris, pers. comm.); the example can be "saved" by applying differential weighting, e.g. with weight 3 for characters $1-3$ and weight 2 for characters $4-5$.
}

$\begin{array}{ccccc}\text { Characters } & \mathbf{a} \mathbf{b} & & \mathbf{a} & \mathbf{b} \\ \text { A } & 00 & \text { A } & 00 ? & 000 \\ \text { B } & 00 & \text { B } & 0 ? 0 & 000 \\ \text { C } & 01 & \text { C } & ? 00 & 11 ? \\ \text { D } & 11 & \text { D } & 111 & 1 ? 1 \\ \text { E } & 11 & \text { E } & 111 & ? 11\end{array}$

FIG. 2. The data set of Fig. 1 in standard (left) and four-taxon (right) representation.

square brackets. For example, $[A B C][D]$ means that either taxa $A, B$, and $C$ have state zero, and taxon $D$ has state one, or vice versa; this statement is uninformative. Conversely, $[\mathrm{AB}][\mathrm{DE}]$ is informative; there are two pairs of taxa that have a different state. We use square brackets to avoid confusion between a four-taxon statement on the one hand and its resolution on a particular cladogram on the other. As an example, the informative four-taxon statement $[A B][C D]$ is accommodated on cladograms that resolve the relationships between taxa $\mathrm{A}, \mathrm{B}, \mathrm{C}$, and $\mathrm{D}$ as, for example, $((A B)(C D))$ or $(D(C(A B)))$ but not on cladograms that resolve the relationships as, for example, $(\mathrm{A}(\mathrm{C}(\mathrm{BD})))$ or $((\mathrm{BC})(\mathrm{AD}))$.

A 0-0-1-1 four-taxon matrix can be derived from the standard representation of the character state distributions in a similar way to the 0-1-1 three-taxon matrix. Such a matrix should include all possible 0-0-1-1 four-taxon statements implied by the standard representation of the character state distributions. An example, using the same taxa and characters as in Fig. 1, is shown in Fig. 2. As with three-taxon statements, the number of informative four-taxon statements implied by a character state distribution depends on the numbers of taxa having the apomorphic state (no) and plesiomorphic state (nz): nz*(nz-1)/2)*(no*(no-1)/2). The number of implied four-taxon statements usually greatly exceeds the number of implied three-taxon statements. Only when $\mathrm{nz}=2$ or $\mathrm{nz}=1$ are there more three- than four-taxon statements (twice as many three-taxon statements for $n z=2$; no implied four-taxon statements at all for $n z=1$ ); when $n z=0, n o=0$, or no $=1$ (no implied three- or four-taxon statements) or when $\mathrm{nz}=3$, there are equal numbers of three- and four-taxon statements.

An individual four-taxon statement does not imply assumptions about polarity, and as a result parsi- 
mony analysis will yield undirected topologies rather than cladograms. As long as the cladogram is not directed, the meaning of an accommodated four-taxon statement $[A B][C D]$ remains equivocal: $A$ and $B$ can be more closely related to each other than either is to $\mathrm{C}$ or $\mathrm{D}$, or $\mathrm{C}$ and $\mathrm{D}$ can be more closely related to each other than either is to A or B; it is only possible to say that at least one or both interpretations must be correct. To obtain cladograms from which hypotheses of polarity can be read, these topologies have to be directed. This is analogous to the situation in standard Fitch or Wagner parsimony, and the same basic possibilities to direct the topologies exist (Nixon and Carpenter, 1993): either good hypotheses about possible outgroup taxa are present or not. In the first case, the four-taxon statements that are considered should include the outgroup taxa as well as the ingroup taxa. After the most-parsimonious topologies for the resulting four-taxon matrix are obtained, they can be rooted between the ingroup and outgroups, if at least the ingroup is monophyletic. If outgroup taxa should appear within the ingroup, the initial assumption of ingroup monophyly is not supported by the data. When there is a lack of good hypotheses for outgroup taxa, one can still fall back on a kind of hypothetical outgroup that reflects a priori assumptions of global plesiomorphy. In this case, only four-taxon statements about the ingroup taxa should be included in the four-taxon matrix. Only after the most-parsimonious topologies are obtained, does the hypothetical ancestor become involved in determining the position of the root by inserting it in the most-parsimonious position. This method of using a hypothetical ancestor is analogous to the situation in the standard approach (Lundberg, 1972; Nixon and Carpenter, 1993).

The fact that the use of three-taxon statements involves stronger a priori assumptions about evolutionary processes than the use of four-taxon statements has an interesting analogy in distance methods (see e.g. Sattath and Tversky, 1977; Farris, 1981): ultrametric distance methods assume that mutation rates are equal among lineages, i.e. that a universal evolutionary clock exists such that all lineages are equally diverged; this strong assumption can be tested a priori by examining if each possible triplet or group of three taxa in the distance matrix satisfies the socalled three-point condition. Conversely, additive distance methods require only that the sum of all branch-lengths between two terminal taxa equals the observed pairwise distance between these taxa. This assumption is less restrictive than the assumption of a universal clock (ultrametric data are, by implication, additive) and it can be tested a priori by examining if each possible quartet of taxa satisfies the so-called four-point condition. Moreover, distance methods exist for data that are approximately additive, which may be said to apply the four-taxon approach to pairwise distance data (Sattath and Tversky, 1977; Fitch, 1981): for each group of four taxa, the observed distances are used to derive a basic unpolarized statement concerning the relationships between these four taxa; in the following step the trees on which the largest number of these basic statements is accommodated are identified.

Kluge (1994: 408-410) presented two hypothetical data sets to illustrate that three-taxon analysis does have problems in finding clades that are supported by reversals only. These can be used to illustrate that four-taxon analysis effectively removes the a priori assumption of irreversibility (the analysis was performed using the computer program ViTA2, which can be obtained from the authors).

Kluge's (1994) first hypothetical matrix (Fig. 3, top) implies a $(\mathrm{FGH}(\mathrm{I}(\mathrm{J} \mathrm{K})))$ clade in which the nested monophyletic groups (I(J K)) are supported by reversals only. This is confirmed in the single mostparsimonious tree found by standard parsimony analysis (Fig. 3, lower left). Three-taxon analysis yields nine most-parsimonious trees, each accommodating 589 out of 708 informative three-taxon statements. From their strict consensus (Fig. 3, lower middle), it is clear that three-taxon analysis considers IJK as a paraphyletic taxon. For four-taxon analysis, there are 1014 informative four-taxon statements for 11 taxa (the hypothetical ancestor is excluded). The single most-parsimonious tree accommodates 964 of these. Adding the hypothetical outgroup in the most-parsimonious way results in the cladogram shown in Fig. 3 (lower right; the same cladogram is obtained when $X$ is considered as a real outgroup taxon; in this case, there are 1722 four-taxon statements for 12 taxa, 1547 of which are accommodated). As expected, (I(J K)) is present in this cladogram. The single difference between the standard and the fourtaxon analysis lies in the fact that four-taxon analysis 
Standard approach

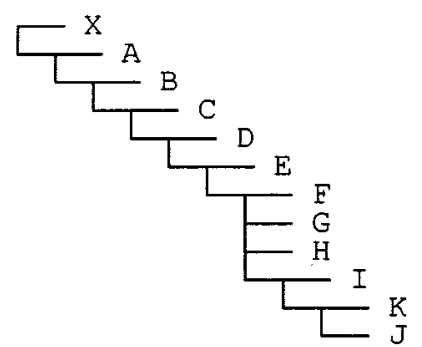

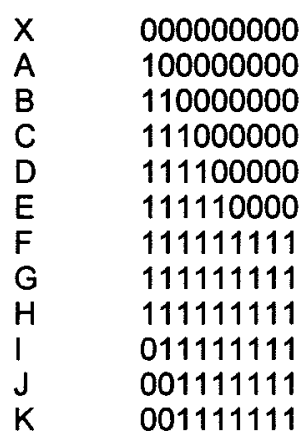

Three-taxon analysis

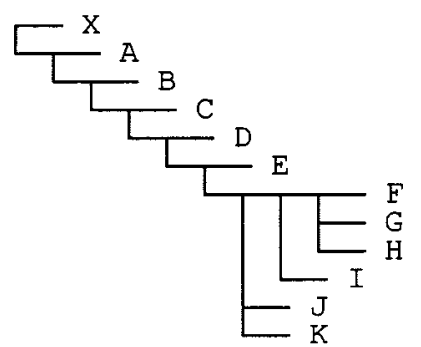

Four-taxon analysis

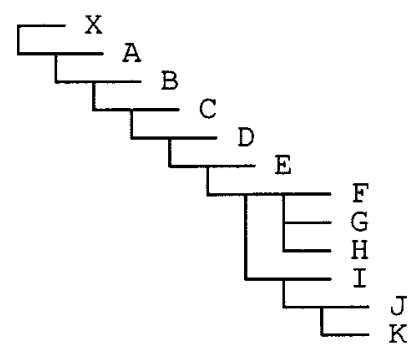

FIG. 3. Kluge's (1993: 408) first hypothetical data set; taxon $X$ is a hypothetical outgroup; reversals in the two first characters specify a clade $(\mathrm{I}(\mathrm{J} \mathrm{K})$ ) that is nested within clade F-K. Left: single most-parsimonious tree in standard parsimony analysis; middle: strict consensus of nine best trees in three-taxon analysis; right: single best tree in four-taxon analysis.

identifies a FGH clade that is unsupported by the data.

Kluge's (1993) second hypothetical data set (Fig. 4, top) contains two highly derived taxa ( $F$ and $G$ ), one of which $(G)$ shows reversal in two characters. In this case, the standard approach and four-taxon analysis give exactly the same result: there is one mostparsimonious tree (Fig. 4, lower left), in which $G$ and

$\begin{array}{ll}\text { X } & 000000 \\ \text { A } & 100000 \\ \text { B } & 110000 \\ \text { C } & 111000 \\ \text { D } & 111100 \\ \text { E } & 111110 \\ \text { F } & 111111 \\ \text { G } & 001111\end{array}$

Standard analysis Four-taxon analysis

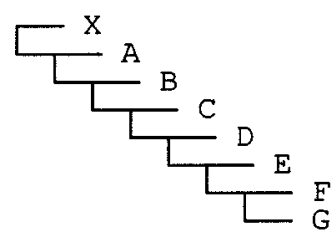

Three-taxon analysis

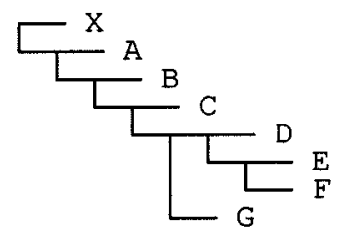

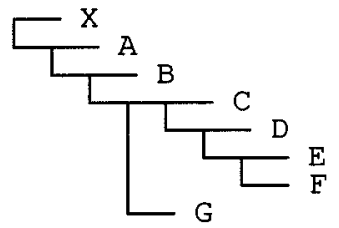

FIG. 4. Kluge's (1993: 408) second hypothetical data set; taxon $X$ is a hypothetical outgroup; $G$ and $F$ are highly derived sister taxa, with $G$ having a reversal in the two first characters, which is confirmed by standard analysis as well as four-taxon analysis (left). Three-taxon analysis (middle and right) identifies two best trees, in both of which $\mathrm{G}$ and $\mathrm{F}$ are far removed from each other. 
F are sister groups. This tree accommodates 56 out of 66 four-taxon statements (or 121 out of 156 when taxon $X$ is considered as a real outgroup taxon). Three-taxon analysis identifies two most parsimonious trees (Fig. 4, lower middle and right), accommodating 67 out of 90 three-taxon statements. In both trees, $G$ is far removed from $F$.

\section{DEPENDENCE AND FRACTIONAL WEIGHTING}

Nelson and Platnick (1991: 363) noted that it might be problematic for the three-taxon approach that not all three-taxon statements implied by a character are logically independent. For example, a character with states 0-1-1-1 for taxa A, B, C, and D implies a total of three three-taxon statements: $\mathrm{A}[\mathrm{BC}], \mathrm{A}[\mathrm{BD}]$, and $A[C D]$. However, because these three statements are derived from the same character, only two of the three are logically independent; whichever two are selected, the third one can be deduced, leaving a ratio of $2 / 3$ of independent to total number of statements ${ }^{2}$. The independency ratio equals $2 / 3$ in this particular case, but it may have different values in other cases. As an example, a character having states $0-0-1-1$ for the same taxa A-D implies only two three-taxon statements, $\mathrm{A}[\mathrm{CD}]$ and $\mathrm{B}[\mathrm{CD}]$ both of which are independent, which gives an independency ratio of $2 / 2$ instead of $2 / 3$. In general, the ratio of independent to total number of statements equals 2 /ot (Nelson and Ladiges, 1992; there are $\mathrm{zt}^{*}(\mathrm{ot} *(\mathrm{ot}-1) / 2)$ three-taxon statements in total, and only $\mathrm{zt}^{*}(\mathrm{ot}-1)$ of them are independent; $\mathrm{zt}$ is the number of taxa having state 0 and ot the number of taxa having state 1).

Nelson and Platnick (1991: 363) suggested compensating for the phenomenon of different ratios of independent statements by reducing the weight of individual statements such that the total weight of all statements derived from a single character is equal to the number of independent statements for that character $^{3}$. This is accomplished by down-weighting all statements by the independency ratio of their character (Nelson and Ladiges, 1992; see also Nelson and Ladiges, 1994). Because the ratio is, by definition, a fraction and because it is applied as a weight, the procedure is called fractional weighting; the ratio is a fractional weight (Nelson and Ladiges, 1992).

\footnotetext{
${ }^{2}$ Similar dependency problems exist in the methods of Sattath and Tversky (1977) and Fitch (1981). These must be added to the inherent dependency problems of distance methods.
}

Character 11100000 for taxa ABCDEFGH: 8 independent statements on a total of $30(z t=5$, ot=3)

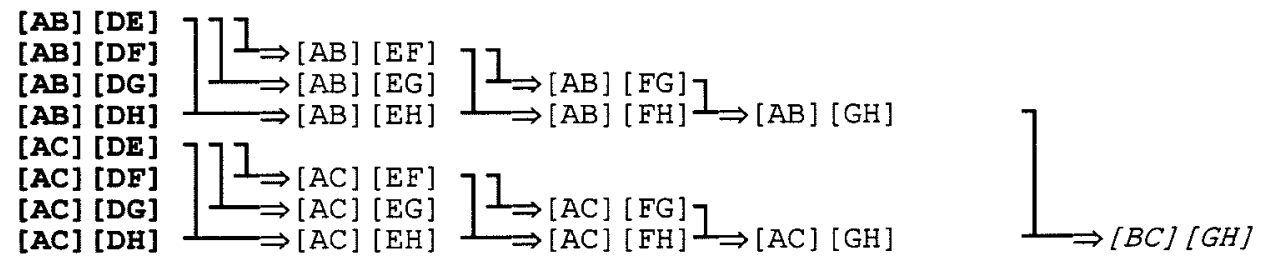

The ten statements of the form $[B C][X Y$ ] result from combining $[A B][X Y]$ and $[A C][X Y]$. This is only shown for $X=G$ and $Y=H$ (italic)

Character 11110000 for ABCDEFGH: 9 independent statements on a total of $36(z t=4, o t=4)$
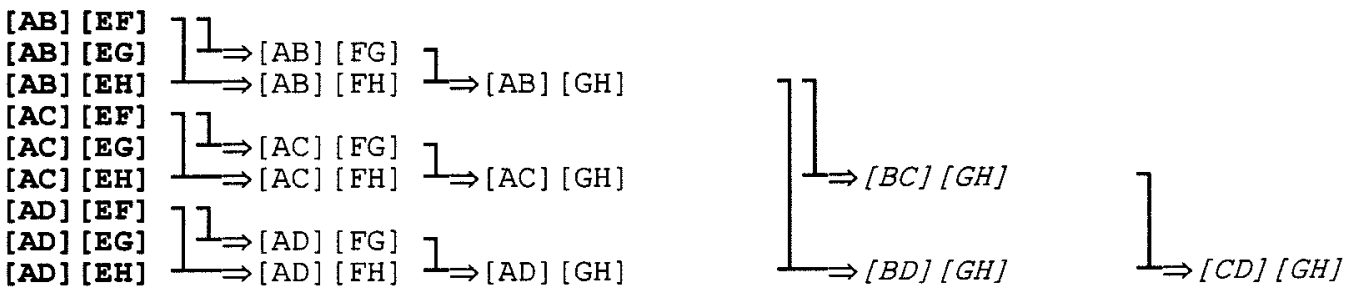

The 18 statements of the form $[B C][X Y],[B D][X Y]$, and $[C D][X Y]$ are obtained as shown for $X=G$ and $\mathrm{Y}=\mathrm{H}$ (italic).

FIG. 5. For any character having zt 0 -taxa and ot 1 -taxa, all $\left(\mathrm{zt}^{*}(\mathrm{zt}-1) / 2\right)^{*}\left(\mathrm{ot}^{*}(\mathrm{ot}-1) / 2\right)$ different implied four-taxon statements can be deduced from any set of $(\mathrm{zt}-1)^{*}(\mathrm{ot}-1)$ independent statements. In the two examples a possible independent set is indicated in bold (left). 
Fractional weighting is easily adapted to four-taxon analysis: the total number of four-taxon statements equals $\left(\mathrm{zt}^{*}(\mathrm{zt}-1) / 2\right)^{*}\left(\mathrm{ot}^{*}(\mathrm{ot}-1) / 2\right)$, and only $(\mathrm{zt}-1) *(\mathrm{ot}-1)$ of them are independent (see Fig. 5 for some examples), which yields the independency ratio $4 /\left(\mathrm{zt}^{*} \mathrm{ot}\right)$. However, the procedure of fractional weighting proposed by Nelson and Ladiges (1992) does not properly solve the problems caused by dependency between basic statements. This is explained below, using three-taxon analysis as an example.

A simple example of fractional weighting is presented in Fig. 6. Character a produces six three-taxon statements, only three of which are independent (a possible choice of independent statements might be $\mathrm{A}[\mathrm{BC}], \mathrm{A}[\mathrm{BD}]$, and $\mathrm{A}[\mathrm{BE}]$; they collectively imply $\mathrm{A}[\mathrm{CD}], \mathrm{A}[\mathrm{CE}]$, and $\mathrm{A}[\mathrm{DE}]$ and are independent among themselves). Character $b$ also yields six statements, but here four out of the six are independent (e.g. $\mathrm{A}[\mathrm{CD}], \mathrm{A}[\mathrm{CE}], \mathrm{B}[\mathrm{CD}]$, and $\mathrm{B}[\mathrm{CE}]$ are indepen-

\footnotetext{
${ }^{3}$ At other places they seem to hold the opinion that logical dependence of statements does not pose a problem; e.g. Platnick (1993) discusses a possible theoretical justification of three-taxon analysis without mentioning the problem, and Nelson (1992: 356), noting that dependency between statements does not alter the data, denied that 'three-taxon analysis produces nonindependent characters' (see Kluge 1994 and Farris et al. 1995 for comments).
}

dent and imply $\mathrm{A}[\mathrm{DE}]$ and $\mathrm{B}[\mathrm{DE}])$. Character c yields three statements $(\mathrm{A}[\mathrm{DE}], \mathrm{B}[\mathrm{DE}]$, and $\mathrm{C}[\mathrm{DE}])$, all three of which are independent. On the single mostparsimonious tree for these data, all three-taxon statements of all three characters are accommodated. When all three-taxon statements are equally weighted, the relative importance of the characters with a low independency ratio is overrated because many of the accommodated statements are not independent. Applying the fractional weights, correctly reduces the relative importance of the characters to their number of independent statements. In this example, the procedure of fractional weighting works correctly because there is no homoplasy in the data set. Indeed, the fractional weights as defined by Nelson and Ladiges (1992) reflect the ratio between independent and total number of accommodated three-taxon statements only in the absence of homoplasy.

This is illustrated by inspecting a second tree for the above data (Fig. 7). On this tree, all three-taxon statements of characters $b$ and $c$ are still accommodated, but in character a there is homoplasy. As a result, $\mathrm{A}[\mathrm{BC}], \mathrm{A}[\mathrm{BD}]$, and $\mathrm{A}[\mathrm{BE}]$ are no longer accommodated, leaving only $\mathrm{A}[\mathrm{CD}], \mathrm{A}[\mathrm{CE}]$, and $\mathrm{A}[\mathrm{DE}]$ as accommodated statements, two of which are independent. Therefore, on this particular tree, the inde-

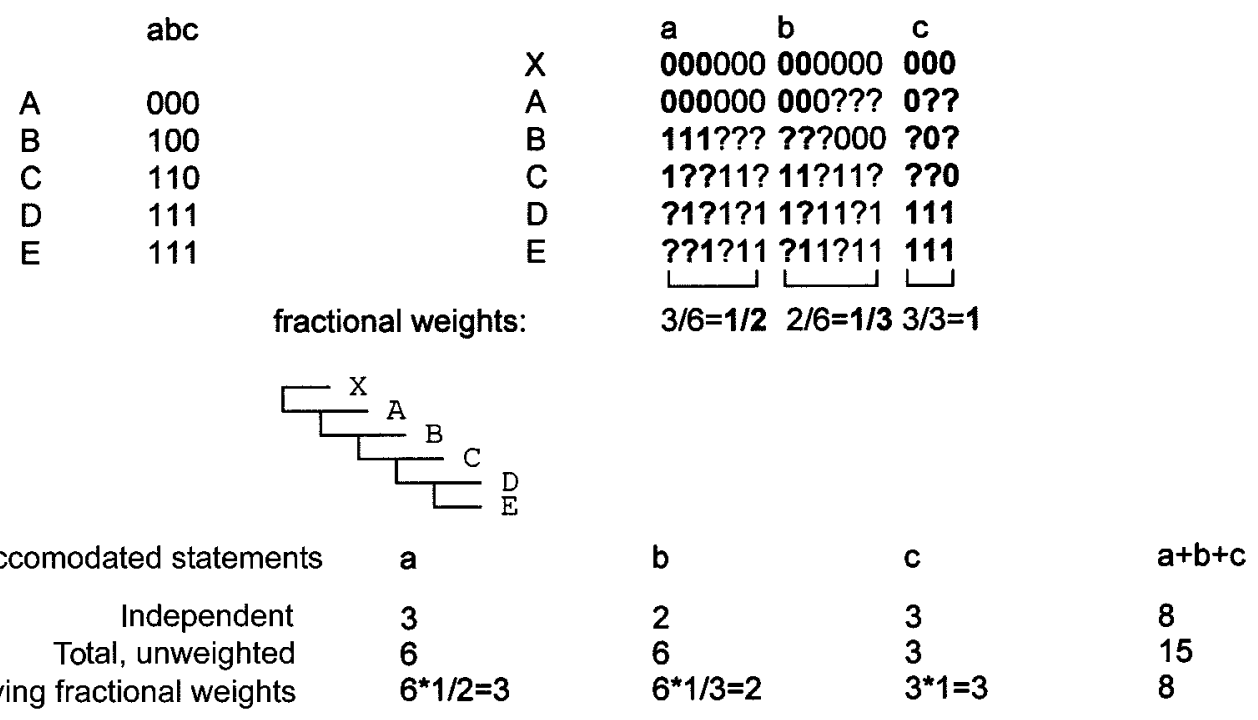

FIG. 6. Hypothetical data set in standard (top left) and three-taxon (top right) representation; for each character a possible choice of independent statements in the three-taxon representation is indicated in bold (left); the single most parsimonious tree has no homoplasy; the total number of fractional weighted accommodated statements equals the total number of independent accommodated statements. 


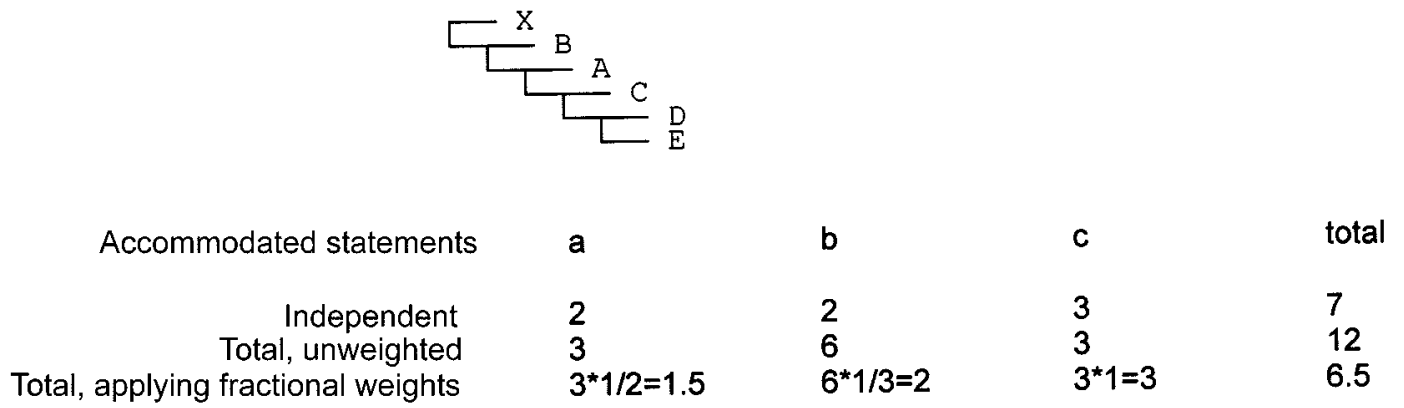

FIG. 7. On this tree fractional weighting underestimates the total number of independent accommodated statements for character a (see Fig. 6 for the character state distributions).

pendency ratio of the accommodated statements for character $a$ is $2 / 3$ instead of $1 / 2$. The fractional weight as defined by Nelson and Ladiges (1992), however, remains fixed at $1 / 2$. As a result, the total number of weighted accommodated statements for this character $\left(3^{*} 1 / 2=1.5\right)$ underestimates the total number of independent accommodated statements (2).

The fact that fractional weighting assumes the lowest possible independency ratio, irrespective of the actual amount of homoplasy on a cladogram, has already been observed by Deleporte (1996: 281-283), who considered it as one of the essential drawbacks of three-taxon analysis compared to standard parsimony. However, because the rationale for doing fractional weighting is to correct for nonindependence of elementary statements (Nelson and Platnick, 1991: 363; Nelson and Ladiges, 1992), it is more accurate to say that fractional weighting does not correctly do what it is supposed to do. From this point of view, an alternative weighting procedure that removes nonindependence correctly only fixes a technical error in the current implementation of three-taxon analysis, and does not alter the basic tenets of the approach.

Even if fractional weighting does not correctly remove nonindependence, it might still be sufficient to identify the trees that accommodate the highest amount of independent basic statements. That it sometimes does identify the correct tree is clear from the previous example; both unweighted and fractional weighted three-taxon analysis consider the tree of Fig. 6 as the best tree for that data set, and that tree is effectively the one that accommodates the highest number of independent three-taxon statements. However, this is not generally true, as shown in the following example.

Consider the data set shown in Fig. 8, assuming that characters $0-5$ each have an a priori weight that is higher than the total number of three-taxon statements implied by characters 6 and 7. Because of these weights and because characters $0-5$ are fully congruent among themselves, the relative positions of taxa $\mathrm{A}-\mathrm{H}$ in the best trees according to the three-taxon approach will be as specified by characters $0-5$. Within these relationships, characters 6 and 7 specify the position of taxon I (Fig. 9; see below for characters 8 and 9): in TREE1, I is the sister group of taxon F (character 6), in TREE 2 it is the sister group of taxon $\mathrm{H}$ (character 7).

Characters 6 and 7 have the same number of 0-taxa and 1-taxa, and therefore the same number of implied three-taxon statements. Also, none of the threetaxon statements of character 7 is accommodated on TREE 1, and none of the three-taxon statements of character 6 is accommodated on TREE2. Therefore, both trees accommodate the same number of threetaxon statements. Any other position of taxon I in TREE 1 beyond that specified in TREE 2 decreases

$\begin{array}{ll} & 01234567 \\ \text { A } & 10000000 \\ \text { B } & 11000000 \\ \text { C } & 11100000 \\ \text { D } & 11110000 \\ \text { E } & 11111000 \\ \text { F } & 11111010 \\ \text { G } & 00000100 \\ \text { H } & 00000101 \\ \text { I } & ? ? ? ? ? ? 11\end{array}$

FIG. 8. A hypothetical data set; cf. Fig. 9. 
the number of accommodated statements for character 6 more than it increases the number of accommodated statements for character 7; conversely, any other position of taxon I in TREE 2 beyond that specified in TREE 1 decreases the number of accommodated statements for character 7 more than it increases the number of accommodated statements for character 6 . Therefore, TREE 1 and TREE 2 are the best three-taxon trees. Because characters 6 and 7 have the same number of 1-taxa, they have the same fractional weight, and both trees are also the best trees under fractional weighting.

Consider two more characters, 8 and 9 (character state distribution shown in Fig. 9), and assume that characters $0-7$ each have an a priori weight that is higher than the total number of three-taxon statements implied by characters 8 and 9 . Under these conditions, the best tree for the enlarged data set will be either TREE 1 or TREE 2 , or both, depending only on the numbers of accommodated statements for characters 8 and 9. The accommodated statements for characters 8 and 9 are listed in Fig. 9, together with a possible set of independent statements. From the summary of these results (Fig. 10), it is clear that both unweighted and fractional weighted three-taxon analysis select TREE 1 as the best tree, even though TREE 2 accommodates one more independent threetaxon statement than does TREE 1 . In the unweighted

TREE 1
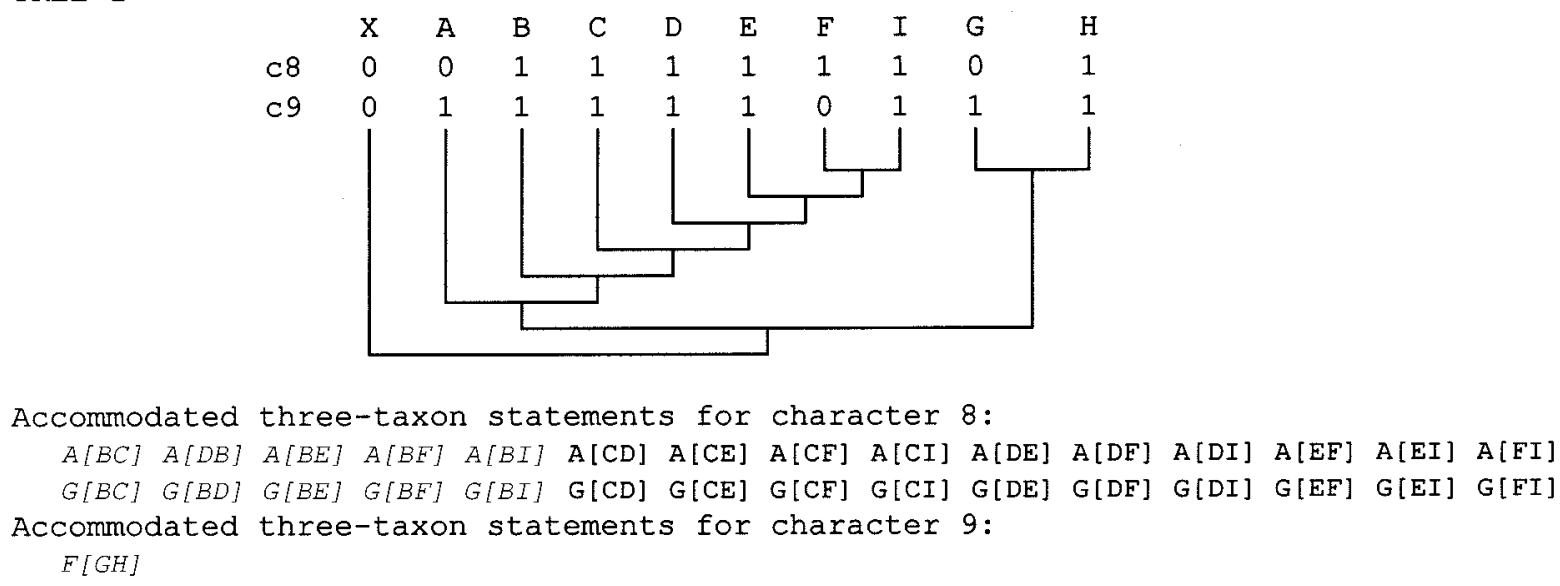

TREE 2
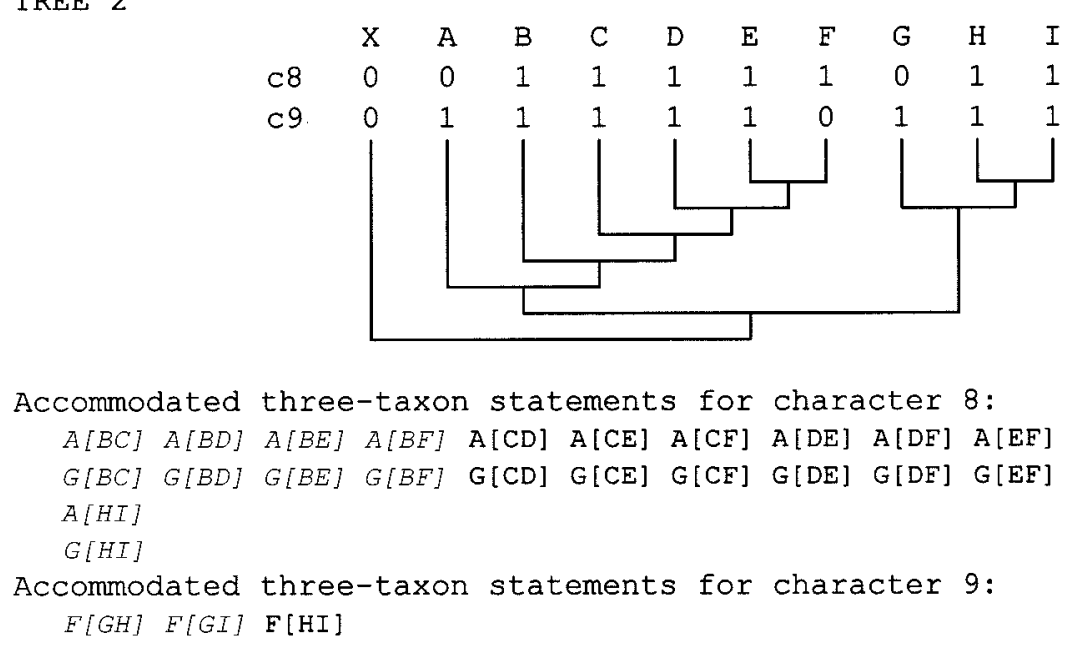

FIG. 9. The two best trees under three-taxon analysis for the data set of Fig. 8 supplemented with characters c8 and c9; the accommodated three-taxon statements for characters c8 and c9 are listed and for each character a possible set of independent accommodated statements is in italics. 
TREE 1

\begin{tabular}{lccl} 
& $\mathrm{t}$ & $\mathrm{t}^{\star} \mathrm{f}$ & $\mathrm{i}$ \\
\cline { 2 - 4 } $\mathrm{c}$ & 30 & 8.57 & 10 \\
$\mathrm{c9}$ & 1 & 0.25 & 1 \\
$\Sigma$ & 31 & 8.82 & 11
\end{tabular}

FIG. 10. Summary of the three-taxon analysis of TREE 1 and TREE 2 (Fig. 9); t: total number of accommodated three-taxon statements; f: fractional weight ( $2 / 7$ for character $\mathrm{c} 8,2 / 8$ for $\mathrm{c} 9)$; i: total number of independent accommodated three-taxon statements.

analysis, TREE 1 is preferred mainly because of the high number of dependent statements in character 8 (20 out of 30). Using fractional weights, the number of independent accommodated statements is underestimated in both cases, but more so in TREE 2 than in TREE1, leading once again to a preference for TREE 1.

This phenomenon also explains the counterintuitive results of three-taxon analysis for some hypothetical data that have been discussed in the literature. As an example, consider the character and the two trees presented by Farris et al. (1995: 213), reproduced here, slightly elaborated, as Fig. 11. Stan- dard parsimony analysis clearly prefers TREE 1 , as this one only requires a single step of homoplasy, compared to five extra steps for TREE2. The same preference is expressed by the numbers of independent accommodated three-taxon statements (216 vs. 168). However, when the total number of accommodated three-taxon statements is taken into account (1080 vs. 1260), the second tree is preferred. As fractional weighting gives the same weight to all statements derived from a single character $(1 / 10$ in this case), it is obvious that the use of these weights will not change this preference (108 vs. 126).

Fractional weighting was introduced as a means of eliminating the distortion produced by different independency ratios for different characters (Nelson and Ladiges, 1992; see also Nelson and Ladiges, 1994). However, based on the above examples, a general conclusion is that it works correctly only in the absence of homoplasy, i.e. when the correct topology is obtained anyhow. Whenever homoplasy is present in a character, the true number of its independent accommodated statements is underestimated, and the degree of underestimation is not related to the number of accommodated independent statements. As a

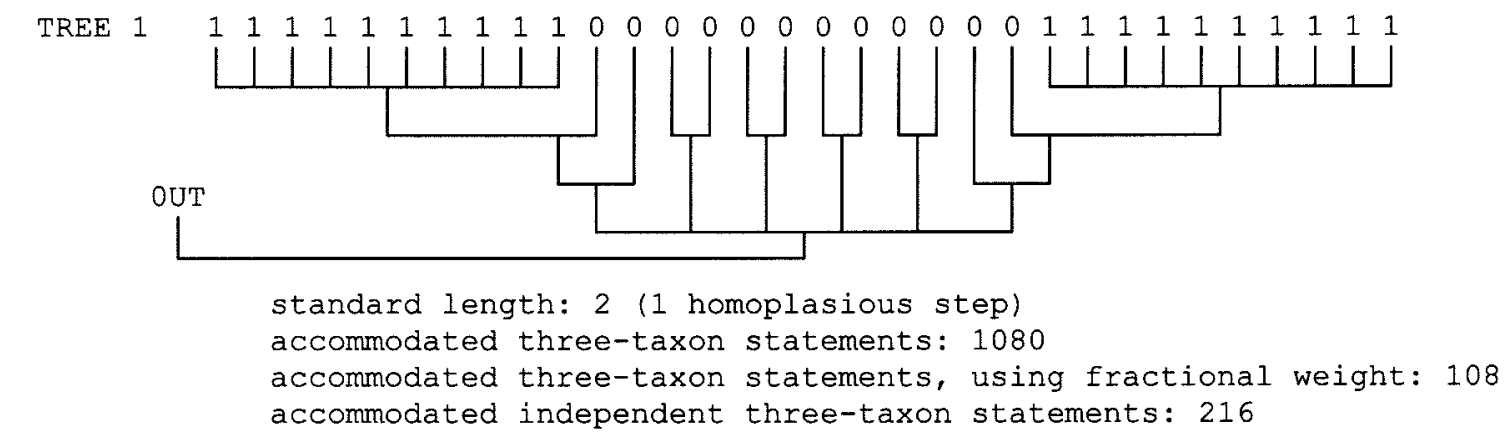

$\begin{array}{llllllllllllllllllllllllllllllllll}\text { TREE } & 2 & 1 & 0 & 0 & 1 & 0 & 0 & 1 & 0 & 0 & 0 & 0 & 1 & 0 & 0 & 1 & 0 & 0 & 1 & 1 & 1 & 1 & 1 & 1 & 1 & 1 & 1 & 1 & 1 & 1 & 1 & 1 & 1\end{array}$

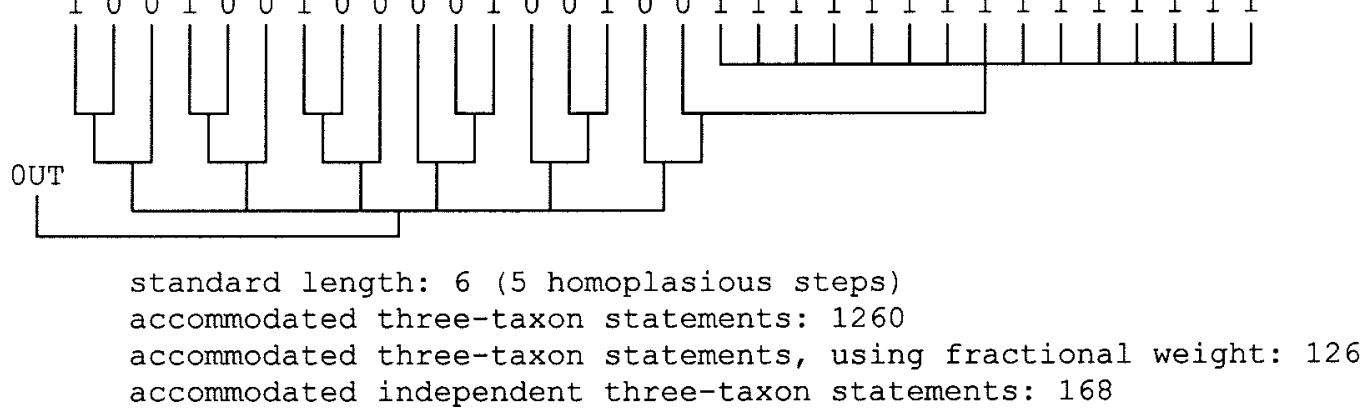

FIG. 11. Farris et al.'s (1995: 213) hypothetical character and tree, showing counterintuitive results when all instead of only the independent accommodated three-taxon statements are taken into account. 
solution to this problem, one might imagine a variation of fractional weighting so that the character's fractional weight is adjusted for each individual tree. However, such an adjusted fractional weight must, by definition, be calculated as the ratio between the number of independent accommodated statements on a particular tree and the total number of accommodated three-taxon statements for the character. So the number of independent accommodated statements has to be known right from the start and the whole procedure of fractional weighting becomes redundant. To correct nonindependence, an algorithm is needed that directly calculates the number of accommodated independent three-taxon statements on a cladogram. As shown for four-taxon analysis by De Laet (1997), such an algorithm can operate directly on the standard representation of a character state distribution (the decomposition of a character state distribution into the implied three- or four-taxon statements is superfluous) and it requires only a single pass over the cladogram.

\section{MUTUAL EXCLUSIVENESS}

In a three-taxon matrix (e.g. Fig. 1), a single informative three-taxon statement $\mathrm{A}[\mathrm{BC}]$ is represented as a binary character that has a zero-entry for taxon $\mathrm{A}$, a one-entry for taxa B and $\mathrm{C}$, and missing entries for all remaining taxa. Harvey (1992: 350) noticed that optimization of these missing entries on a cladogram may lead to assignment of wrong states to those remaining taxa. A simple example is presented in Fig. 12. Three-taxon statement $\mathrm{A}[\mathrm{CE}]$ is accommodated on the tree shown; only a single character state transition is required to explain the character state distribution of the statement. However, in order to arrive at that single step, one has to assume: (1) that the three inner nodes between $C$ and $E$ have state 1 and the remaining inner node has state 0 ; and (2) that the question

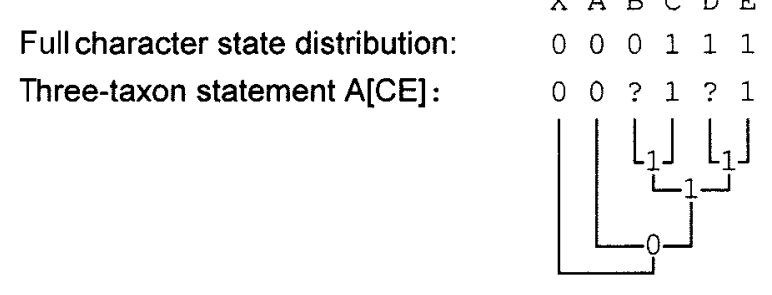

FIG. 12. Accommodated three-taxon statement $A[C E]$ wrongly assumes state 0 for taxon $\mathrm{B}$.

marks for taxa B and D represent state 1. The latter is problematic for taxon $B$, in which state 0 was observed. Both Nelson and Platnick (e.g. Nelson, 1992: 358; Platnick, 1993: 267) replied that optimizations of these missing entries are completely irrelevant: the missing entries in a column of a three-taxon matrix are inserted merely to be able to use widespread computer programs for testing if the corresponding three-taxon statement is accommodated or not.

However, Farris et al. (1995) reformulated the problem such that optimizations of the missing entries are no longer the issue. They pointed out that an accommodated three-taxon statement $\mathrm{A}(\mathrm{BC})$ gets its evidential value from the fact that the presence of character state 1 in taxa B and C can be explained by inheritance of state 1 from a common ancestor that is not shared with taxon $\mathrm{A}$, which puts a restriction on the character states that can possibly be assigned to the inner nodes of the cladogram. This in turn can lead to contradictions when different accommodated statements of a single character are compared.

A simple case, using the same character and the same tree as in Fig. 12, is presented in Fig. 13. On this tree, four out of the six available three-taxon statements are accommodated: $\mathrm{B}[\mathrm{DE}], \mathrm{A}[\mathrm{CD}], \mathrm{A}[\mathrm{CE}]$, and $A[D C]$. Since $A[D E]$ follows from $A[C D]$ and $A[C E]$ only three of them are independent. First consider statement $\mathrm{B}[\mathrm{DE}]$. Without reference to a tree, this statement hypothesizes that the presence of the ple-
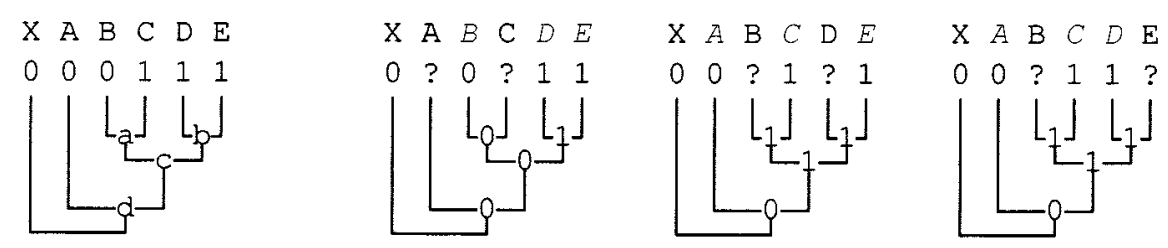

FIG. 13. Accommodated three-taxon statements $B[D E]$ on the one hand and $A[C E]$ or $A[C D]$ on the other assign conflicting states to inner nodes a and $\mathrm{c}$ when they are explained by common descent. 
siomorphic state in B and the apomorphic state in D and $\mathrm{E}$ is an indication that $\mathrm{D}$ and $\mathrm{E}$ are more related to each other than either is to $B$. The fact that the statement is accommodated on the tree is taken as an indication that the tree supports the hypothesis because the origin of the derived state can be traced back to an ancestor of $\mathrm{D}$ and $\mathrm{E}$ that is not an ancestor of $B$ (in this case to the stem species at inner node b); taxon $B$ has retained the plesiomorphic condition that is present at stem species a and c. When the same reasoning is applied to statements $A[C D]$ or $A[C E]$ a problem arises: starting from $\mathrm{C}$ and either $\mathrm{D}$ or $\mathrm{E}$, the origin of the derived state is traced back through nodes $a$ and $b$ to node $c$. So in order to explain $A[C D]$ or $\mathrm{A}[\mathrm{CE}]$ by common descent it has to be assumed that nodes a and $c$ have the apomorphic state, but explaining $\mathrm{B}[\mathrm{DE}]$ by common descent required that nodes a and c have the plesiomorphic state. Since all three statements concern the same character, these results are mutually exclusive; the statements that can be explained by common descent on the cladogram are either both $\mathrm{A}[\mathrm{CD}]$ and $\mathrm{A}[\mathrm{CE}]$ or $\mathrm{B}[\mathrm{DE}]$, but not all three simultaneously.

Platnick et al. (1996) argued that this kind of contradiction is not relevant because it follows from the premise that plesiomorphies require explanation, which, in their opinion, is faulty because it comes down to grouping by plesiomorphy. However, this is based on a confusion of concepts. Farris et al.'s (1995) level of explaining character states recognizes a lack of historical contiguity in character states (Deleporte, 1996; De Laet and Smets, 1998). They pointed out that such an explanation of the distribution of one character state has purely logical consequences for the explanation of the distribution of the alternative character state. Whatever other consequences this may have, it does not lead to grouping by plesiomorphy. Group recognition is logically independent of discovering historical noncontiguity of character states and depends on the direction imposed on a most-parsimonious topology, which is mostly done by outgroup comparison (see Nixon and Carpenter, 1995). As discussed above, three-taxon analysis, as proposed by Nelson and Platnick (1991), is closely tied to the notion of irreversibility of character evolution, but even then the distinction holds.

According to another line of reasoning (Platnick in Platnick et al., 1996), a three-taxon statement is a higher level statement about relationships that is not concerned with character state distributions, and as a result the above objections of Farris et al. (1995) are not relevant. However, this argument overlooks the fact that higher level statements about relationships must also somehow be based on empirical observation, or at least it fails to make the connection between observation and higher level statement clear. Even if it is accepted that a single three-taxon statement is based on empirical observation, the logical basis of accepting a cladogram that accommodates the highest number of such statements as the best hypothesis concerning relationships is still far from clear. In standard parsimony the levels and their connections are clear: (1) empirical observation leads to hypotheses of primary homology, i.e. the circumscription of characters (cf. Platnick, 1979: 542), with each character being a crude hypothesis of relationships; (2) parsimony analysis leads to undirected topologies consistent with the maximum possible historical contiguity of character states over all available characters; (3) direction of these topologies leads to hypotheses of relationships that are based on all available evidence simultaneously.

Farris et al. (1995) only discussed three-taxon analysis, but it is clear that the criticism applies equally well to four-taxon analysis. This is easily demonstrated by reconsidering the previous example, this time assuming that the hypothetical outgroup $X$ in Fig. 13 is instead a real taxon. In that case, $[\mathrm{XB}][\mathrm{DE}],[\mathrm{XA}][\mathrm{CD}]$ and $[\mathrm{XA}][\mathrm{CE}]$ are a largest set of independent accommodated four-taxon statements. As in the previous example, $[\mathrm{XB}][\mathrm{DE}]$ on the one hand and $[X A][C D]$ and $[X A][C E]$ on the other are mutually exclusive with respect to implied character states for the inner nodes. If the rationale for fourtaxon analysis is to maximize the number of independent basic statements that can be explained by common descent, then only [XA][CD] and [XA][CE] should be accepted as accommodated statements, and $[\mathrm{XB}][\mathrm{DE}]$ rejected (alternative solutions may be possible; for example by simultaneously taking into account multiple sets of state assignments to inner nodes comparable to what happens in maximum likelihood approaches; or by allowing polymorphic inner nodes, as in Farris (1978) or Felsenstein (1979); or by simply accepting all three statements and considering the approach as a phenetic method for 
character data, grouping ahistorically on overall similarity). Accommodated statements that do not exclude each other, such as [XA][CD] and [XA][CE], will be referred to as compatible statements because they are compatible with the same set(s) of inner node state assignments. The best trees according to four-taxon analysis are then those trees that maximize the number of compatible independent accommodated four-taxon statements for the data available. Because the number of compatible independent accommodated statements for a given character on a given cladogram depends upon the states that are assigned to the inner nodes, any algorithm to calculate the maximum number must, at the same time, calculate the inner node assignments that achieve this maximum. In order to discuss this optimality criterion, it is useful to discuss an alternative formulation of standard parsimony first.

\section{STANDARD PARSIMONY AS TWO-TAXON ANALYSIS}

Assume a binary character that has zt 0 -taxa and ot 1-taxa. A priori this character state distribution hypothesizes that there are $\mathrm{zt}-1$ independent pairwise similarities between taxa that have character state zero for this character, and ot-1 independent pairwise similarities between taxa that have state one. First consider a tree on which the character has no homoplasy. Any such tree can be subdivided into two parts, a first in which all inner nodes have state 0 , and a second in which all inner nodes have state 1; the branch between both parts of the tree is the branch along which the single necessary state transition occurs. As a result, all (ot -1$)+(\mathrm{zt}-1)$ a priori pairwise similarities are accommodated, and the tree is consistent with the explanation that all (ot -1$)+$ $(\mathrm{zt}-1)$ pairwise similarities are due to common descent. Next assume a tree on which a most parsimonious reconstruction requires one step of homoplasy. Any most parsimonious reconstruction on any such cladogram implies two state transitions, and these two state transitions subdivide the tree into three parts: either one part in which all nodes have state 0 and two parts in which all nodes have state 1, or two parts in which all nodes have state 0 and one part in which all nodes have state 1 ; in the first case there is precisely one independent pairwise similarity between 1-taxa that can no longer be explained by common descent, in the second case there is precisely one independent pairwise similarity between 0-taxa that can no longer be explained by common descent. Which one of the two possibilities occurs depends on the particular tree and on the particular mostparsimonious reconstruction chosen, but in both cases there is exactly one independent pairwise similarity that can no longer be explained by common descent. In a similar way, any subsequent step of homoplasy will imply that one more independent pairwise similarity can no longer be explained by common descent. In summary, if $\mathrm{h}$ represents the amount of homoplasy in a character on a tree, then the amount of a priori pairwise similarities that can still be explained by common descent is equal to $(n z-1)+$ $($ no -1$)-\mathrm{h}^{4}$. Similar reasoning can be applied to multistate characters. From this point of view, standard parsimony analysis searches for the tree(s) on which the highest number of compatible independent pairwise a priori similarities can be accommodated, and as such it can be characterized as two-taxon analysis (De Laet, 1997).

Note that the above argument is basically the complement of Farris' (1983: 20, 22; see also Farris et al. 1995: 212-213) argumentation to count independent ad hoc hypotheses of homoplasy (it stresses independent pairwise similarities rather than independent pairwise homoplasies, as Farris did). To illustrate this, consider Farris' (1983: 20) example of 20 taxa that share a feature and a putative genealogy that assigns these taxa to two distinctly related groups $\mathrm{A}$ and B of 10 taxa each:

\footnotetext{
"There are 100 distinct two-taxon comparisons of members of A with members of $B$, and each of those similarities in $X$ considered in isolation comprises a homoplasy. Those homo-
}

\footnotetext{
${ }^{4}$ Farris (1983: 13) showed that (we quote Platnick, 1993: 271) "cladistics is agnostic about the status of characters parsimoniously optimized as synapomorphies (i.e. they may be, but are not necessarily, true marks of history), requiring, as a method, only the conclusion that each incongruent character not be a true mark of history". The current argument refines this to the level of individual independent pairwise similarities and their explanation in terms of historical contiguity: each step of homoplasy requires that there is one independent similarity for which the explanation by historical contiguity is refuted; nothing definite is implied about the status of the remaining similarities.
} 
plasies do not constitute independent required hypotheses, however. The genealogy does not require that similarities in $\mathrm{X}$ within either group be homoplasies; it is consistent with identity by descent of $X$ within each group. If $X$ is identical by descent in any two members of $\mathrm{A}$, and also in any two members of $\mathrm{B}$, then the A-B similarities are all homoplasies if any one of them is. The genealogy thus requires but a single ad hoc hypothesis of homoplasy"

Similarly, there are $10 * 9 / 2=45$ distinct two-taxon comparisons of members of $A$, and as much pairwise comparisons of members of $\mathrm{B}$; the genealogy is consistent with identity by descent of $X$ in each of these 90 pairwise comparisons. In each group, however, only 9 out of the 45 are logically independent. So from the total of 19 independent a priori similarities in $X, 9$ can be explained by common descent in group $\mathrm{A}$ and another 9 by common descent in group B, leaving only a single pairwise independent similarity in $\mathrm{X}$ that cannot be explained by common descent.

The optimality criterion for four-taxon analysis can be formulated in a similar way: just as standard parsimony, four-taxon analysis searches for the tree(s) on which the highest number of compatible independent pairwise similarities can be accommodated, but there is one restriction: for any character, accommodated similarities are only counted if the implied reconstruction of inner nodes accommodates both accommodated similarities between 0-taxa and accommodated similarities between 1-taxa at the same time (De Laet, 1997). As a result, four-taxon analysis will lead to reconstructions of inner nodes that are also found in standard parsimony except when no most parsimonious reconstruction (sensu Swofford and Maddison, 1987) exists that has both accommodated zero- and one-similarities. In that case fourtaxon analysis will select a non-most-parsimonious reconstruction that, at the same time, accommodates zero- and one-similarities, at least if such a reconstruction exists. In other words, whenever possible four-taxon analysis avoids reconstructions of inner nodes that imply that all similarities for a given character state are due to homoplasy. As a result, four-taxon analysis may lead to very counterintuitive hypotheses concerning the evolution of characters, as illustrated in the following examples.

For a given tree and set of inner node state assignments, let IASZ denote the number of independent accommodated similarities between taxa that have state 0 , and IASO the number of independent accommodated similarities between taxa that have state 1 . Whenever either IASZ or IASO drops to 0 (e.g. Fig. 14, left; the set of inner node state assignments STA1 specifies a most-parsimonious reconstruction), the number of accommodated four-taxon statements also drops to 0 . Therefore any set of inner node state assignments that manages to keep both IASZ and IASO greater than 0 will accommodate more statements and should be preferred (e.g. Fig. 14, right; the set of inner node state assignments STA2 is not a
STA1

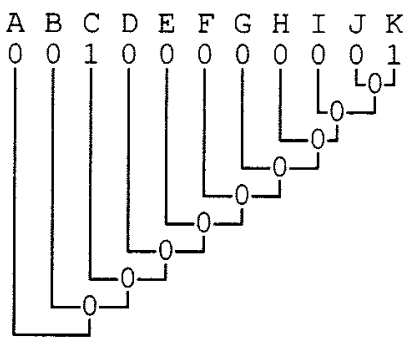

STA2

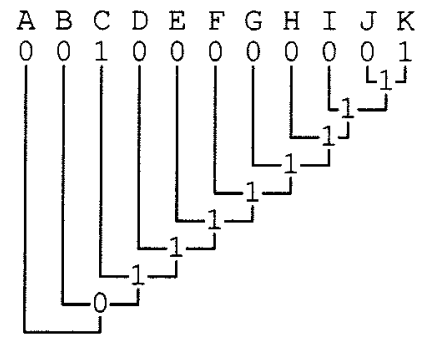

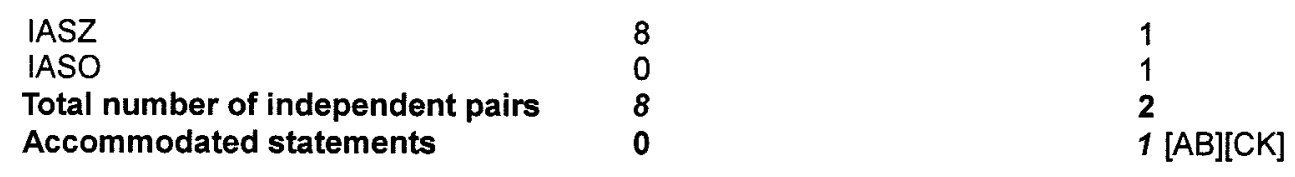

FIG. 14. The inner node state assignments that accommodate the highest number of independent pairwise similarities (STA1) on a given tree are not necessarily those that accommodate the highest number of independent accommodated four-taxon statements (STA2). IASZ: independent accommodated similarities between taxa having state 0; IASO: independent accommodated similarities between taxa having state 1. 
most-parsimonious reconstruction). The accommodation of the single accommodated statement [AB][CK] on the tree therefore requires that the set of inner node state assignments STA2 is preferred over STA1, which in turn implies that character state 0 arose independently in each of the seven lineages leading to taxa D-J. STA1, conversely, requires that character state 1 arose independently in two lineages only; but accepting this reconstruction implies that $[\mathrm{AB}][\mathrm{CK}]$ is no longer accommodated. The pectinate series D-J can be extended ad libitum, making the implications for the evolution of the character under four-taxon analysis increasingly unrealistic.

A related effect is illustrated in Fig. 15, where two different trees are compared (the two trees differ only in the positions of taxa B and C). With the inner node state assignments as shown in TREE 1 , one four-taxon statement is accommodated; this is the best possible result for this tree: any other assignments lead to a loss of the accommodated statement. However, in order to accommodate this statement, it has to be assumed that state 0 arose independently in each of the seven lineages leading to one of the taxa D-J. On TREE 2, on the other hand, no four-taxon statements can be accommodated, whatever the inner node state assignments. Therefore, four-taxon analysis of this tree does not forbid the explanation that character state 1 arose independently in the two lineages leading to taxa $\mathrm{C}$ and $\mathrm{K}$. In terms of accommodated statements, TREE 1 is only slightly better than TREE 2 , but it necessitates unrealistic hypotheses about the evolution of the character.

The restriction that for each character both IASZ and IASO should exceed 0 whenever possible has no obvious biological interpretation. It is a methodological constraint of four-taxon analysis as defined thus far and follows from the basic tenet distinguishing the three-taxon approach from the standard approach to parsimony analysis: character state distributions should be broken up into the smallest possible set of statements that are each still informative with respect to relationships. So let us have a closer look at this tenet.

\section{THE NATURE OF SYSTEMATIC DATA}

Nelson and Platnick have repeatedly raised the question of what constitutes systematic data. Nelson (1993: 261), for example, wonders if empirical observation yields either binary characters as represented in a conventional matrix or three-taxon statements, while Platnick (1993: 271) discusses the possibility

\footnotetext{
"that the smallest relevant observation about character diversity is not a single entry in a character $x$ taxon matrix, but a homology statement, which must minimally be a three-taxon statement homologizing a feature found in two out of three taxa"
}

However, systematic data arise neither as fully developed binary characters nor as three-taxon statements, and the smallest relevant observation about character diversity is neither a single entry in a standard matrix nor a three-item statement.

Consider a binary character. As a whole, such a character expresses the hypothesis or theory that
TREE 1

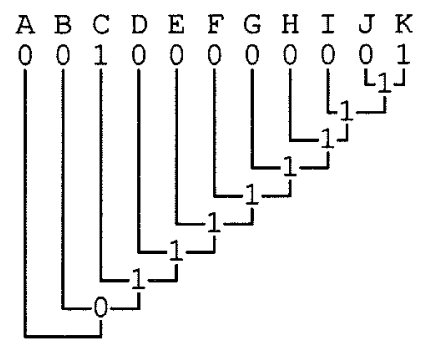

TREE 2

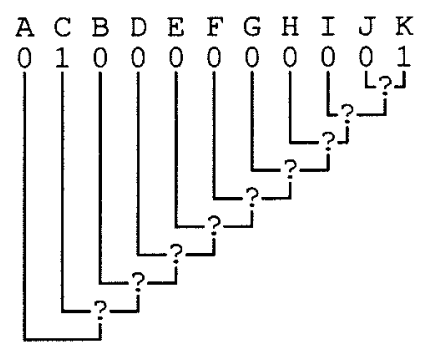

\section{Accommodated Statements}

$1[\mathrm{AB}][\mathrm{CK}]$

FIG. 15. A marginal increase in the number of accommodated four-taxon statements may impose strong restrictions on possible inner node states. 
"two attributes which appear different in some way are nonetheless the same (or homologous)" (Platnick 1979: 542; see also de Pinna 1991).

Similar, but lower-level, theories are implied for each of both attributes because the precise expression of a single attribute may vary in different taxa (Platnick 1979). The samenesses expressed in the high-level and the two lower-level theories are variously called similarities, topological correspondences or primary homologies, and the various means by which hypotheses of primary homology can be established are often referred to as homology criteria (Rieppel, 1988; de Pinna, 1991). The high level theory expresses the a priori expectation that at a certain hierarchical level, the group of all taxa possessing one or the other of both attributes is monophyletic. It is implied that all possible modifications of the attribute as present in the stem species of this monophyletic group are covered by the two lower-level theories. These lower-level theories furthermore express the a priori expectations that within the monophyletic group (1) all taxa possessing the first attribute are in historical contiguity; and (2) all taxa possessing the second attribute are also in historical contiguity. These expectations are the hypotheses tested during parsimony analysis (cf. de Pinna 1991). Note that the prior expectation for the lower level hypotheses is historical contiguity rather than monophyly because the character does not entail a priori polarity decisions. Precisely by not deciding a priori if the second attribute is a modification of the first (leading to a broader conception of the first), or vice versa (leading to a broader conception of the second), or if both are modifications of an unobserved ancestral state, statements of that kind become amenable to testing by means of congruence during parsimony analysis.

Assume a binary character and a set of taxa, nz of which have the first attribute (state 0 ), and no the second (state 1). What then happens when a next taxon with a comparable feature (i.e. covered by the high level theory) is taken into consideration? On the basis of the $\mathrm{nz}+$ no taxa that have been examined before, we will no doubt have developed a general conception of both attributes (which might be called their essences), and it may appear that scoring a new taxon comes down to simply checking if an attribute in the new taxon conforms to either of both conceptions; if this is not the case, a third state may be conceived of, turning the character into a multistate character. This point of view is succinctly summarized in the idea that the smallest relevant observation about character diversity is a single entry in a character $\mathrm{x}$ taxon matrix. Alternatively, one could develop the general conception of both attributes directly on the basis of the complete group of taxa, including the new one. From this point of view, one could say that systematic data arise as fully developed characters.

Although both points of view may be correct as higher level descriptions, more basic operations and decisions lie underneath. If on the basis of a homology criterion it is decided that a new taxon has the first rather than the second attribute, this hypothesis is a compound statement that serves as a convenient summary of the elementary homology decisions in all possible pairwise comparisons between the new taxon on the one hand and the taxa that have been examined before on the other. Indeed, the new taxon will be scored 0 only if: (1) in each pairwise comparison between the new taxon and a taxon that has previously been scored as zero, the feature being compared is considered homologous under the homology criteria that are used; and (2) in each pairwise comparison between the new taxon and a taxon that has previously been scored as 1 , the feature being compared is considered nonhomologous under the homology criteria that are used. The level of homology and nonhomology in these pairwise comparisons is obviously the lower level discussed above, and implies the prior expectation of historical contiguity rather than monophyly. All other outcomes concerning the prior status of the feature in the new taxon can likewise be expressed in terms of these elementary pairwise homology statements. Summarizing, given a higher level theory that homologizes two attributes (coded as 0 and 1) with respect to an implicit nonhomologous third attribute, the smallest relevant observation with respect to character diversity is the theory that a feature found in two taxa is homologous (lower level). It is expected that this lower level homology can be explained by historical contiguity, and as discussed above, standard parsimony identifies the tree(s) that accommodate(s) the maximum number of such logically independent prior expectations over all characters of a data set. The two-taxon statements are relevant precisely because 
they can be either corroborated or refuted by confronting them with such statements about other characters; they are the smallest possible relevant statements because statements about only a single taxon can never be refuted by means of such confrontation.

From this point of view, a four-taxon statement is a compound statement that entails two elementary relevant statements. In four-taxon analysis, these are coupled such that the four-taxon statement as a whole is only accepted if both composing elementary statements are accepted simultaneously, which leads to counterintuitive results such as presented in Figs 14 and 15. Such anomalous results can now easily be explained: four-taxon analysis correctly considers a rejected four-taxon statement as a false mark of history, but it does not take into account that two degrees to which a rejected statement can be false exist: either one or both of the implied elementary statements can be false marks of history. The conclusion is that four-taxon analysis is not a valid method for biological systematics.

\section{NOTE ADDED IN PROOF}

After submission of our paper, Farris (1997) published a most incisive critique of three-taxon analysis. Besides discussing some issues that are not dealt with in this paper, such as Platnick et al.'s (1996: 249) call for congruence studies (Farris 1997: 140), Farris deals at length with the problems reversals pose for three-taxon analysis (Farris 1997: 135-140; see our section on irreversibility) and with the function of optimization in standard parsimony analysisexplaining similarities by inheritance (Farris 1997: 132-133; see our section on mutual exclusiveness). Farris' and our treatments, partly overlapping and partly complementary, are congruent in both cases. With regard to explaining similarities by inheritance, Farris (1997: 133) points out a contradiction that we did not notice in Platnick's attempts to justify threetaxon analysis: Platnick (1993: 271) argues that Farris' (1983) classic justification of standard parsimony analysis, including explanation of accommodated similarities by the common cause of ancestry, applies equally well to three-taxon analysis; yet at the same time he rejects character optimization and the notion of ancestral character states as applied to three-taxon statements (Platnick in Platnick et al., 1996), even though their only function is to assure a logically correct explanation by common descent.

\section{REFERENCES}

Camin, J. H., and Sokal, R. R. (1965). A method for deducing branching sequences in phylogeny. Evolution 19, 311-326.

De Laet, J. (1997). "A Reconsideration of Three-Item Analysis, the Use of Implied Weights in Cladistics, and a Practical Application in Gentianaceae." Ph.D. thesis, Katholieke Universiteit Leuven.

De Laet, J., and Smets, E. (1995). Four-item analysis: an undirected implementation of the three-item approach. XIV Meeting of the Willi Hennig Society, Abstracts: 3 . College Station, Texas.

De Laet, J., and Smets, E. (1998). On the TTSC-FTSC formulation of standard parsimony. Cladistics 14, 239-248.

Deleporte, P. (1996). Three-taxon statements and phylogeny reconstruction. Cladistics 12, 273-289.

de Pinna, M. C. C. (1991). Concepts and tests of homology in the cladistic paradigm. Cladistics 7, 367-394.

Farris, J. S. (1978). Inferring phylogenetic trees from chromosome inversion data. Syst. Zool. 27, 275-284.

Farris, J. S. (1981). Distance data in phylogenetic analysis. In "Advances in Cladistics. Proceedings of the First Meeting of the Willi Hennig Society". (V. A. Funk, and D. R. Brooks, Eds), pp. 3-23. New York Botanical Garden, Bronx, New York.

Farris, J. S. (1983). The logical basis of phylogenetic analysis. In "Advances in Cladistics Vol. 2. Proceedings of the Second Meeting of the Willi Hennig Society". (N. Platnick, and V. A. Funk, Eds), pp. 7-36. Columbia University Press, New York.

Farris, J. S. (1988). “Hennig86. Version 1.5”. Program and Documentation. Port Jefferson Station, New York.

Farris, J. S. (1997). Cycles. Cladistics 13, 131-144.

Farris, J. S., Källersjo, M., Albert, V. A., Allard, M., Anderberg, A., Bowditch, B., Bult, C., Carpenter, J. M., Crowe, T. M., De Laet, J., Fitzhugh, K., Frost, D., Goloboff, P., Humphries, C. J., Jondelius, U., Judd, D., Karis, P. O., Lipscomb, D., Luckow, M., Mindell, D., Muona, J., Nixon, K., Presch, W., Seberg, O., Sidall, M. E., Struwe, L., Tehler, A., Wenzel, J., Wheeler, Q., and Wheeler, W. (1995). Explanation. Cladistics 11, 211-218.

Felsenstein, J. (1979). Alternative methods of phylogenetic inference and their interrelationship. Syst. Zool. 28, 49-62.

Fitch, W. M. (1971). Towards defining the course of evolution: minimum change for a specific tree topology. Syst. Zool. 20, 406-416.

Fitch, W. (1981). A non-sequential method for constructing trees and hierarchical classifications. J. Mol. Evol. 18, 30-37.

Goloboff, P. A. (1993). "NONA version 1.1." Program and Documentation. Distributed by the author.

Harvey, A. W. (1992). Three-taxon statements: more precisely, an abuse of parsimony. Cladistics 8, 345-354.

Kluge, A. G. (1993). Three-taxon transformations in phylogenetic 
inference: ambiguity and distortion as regards explanatory power. Cladistics 9, 246-259.

Kluge, A. G. (1994). Moving targets and shell games. Cladistics 10, 403-413.

Kluge, A. G., and Farris, J. S. (1969). Quantitative phyletics and the evolution of Anurans. Syst. Zool. 18, 1-32.

Ladiges, P. Y., Prober, S. M., and Nelson, G. (1992). Cladistic and biogeographic analysis of the 'blue ash' Eucalypts. Cladistics 8, 103-124.

Lundberg, J. G. (1972). Wagner networks and ancestors. Syst. Zool. 21, 398-413.

Morrone, J. J., and Carpenter, J. M. (1994). In search of a method for cladistic biogeography: an empirical comparison of component analysis, Brooks parsimony analysis, and three-area statements. Cladistics 10, 99-153.

Nelson, G. (1992). Reply to Harvey. Cladistics 8, 355-360.

Nelson, G. (1993). Reply. Cladistics 9, 261-265.

Nelson, G. (1994). Homology and systematics. In "Homology: the hierarchical basis of comparative biology". (B. K. Hall, Ed.), pp. 101-149. Academic Press, San Diego.

Nelson, G. (1996). Nullius in verba. Published by the author.

Nelson, G., and Platnick, N. I. (1991). Three-taxon statements: a more precise use of parsimony. Cladistics 7, 351-366.

Nelson, G., and Ladiges, P. Y. (1991a). Three-area statements: standard assumptions for biogeographic analysis. Syst. Zool. 40, 470-485.

Nelson, G., and Ladiges, P. (1991b). Standard assumptions for biogeographic analysis. Austr. Syst. Bot. 4, 41-58. (addendum; 5, 247).

Nelson, G., and Ladiges, P. (1992). Information content and fractional weight of three-item statements. Syst. Biol. 41, 490-494.

Nelson, G., and Ladiges, P. Y. (1993). Missing data and three-item analysis. Cladistics 9, 111-113.

Nelson, G., and Ladiges, P. Y. (1994). Three-item consensus: empirical test of fractional weighting. In "Models in phylogeny reconstruction". (R. W. Scotland, D. J. Siebert, and D. M. Williams, Eds), pp. 193-209. Oxford University Press, Oxford.

Nelson, G., and Ladiges, P. Y. (1995). “TAX: MSDos Computer
Programs for Systematics". Program and Documentation. Published by the authors.

Nelson, G., and Ladiges, P. Y. (1996). Paralogy in cladistic biogeography and analysis of paralogy-free subtrees. American Museum Novitates 3167, 1-58.

Nixon, K. C., and Carpenter, J. M. (1993). On outgroups. Cladistics 9, 413-426.

Patterson, C., and Johnson, G. D. (1995). The intermuscular bones and ligaments of telostean fishes. Smiths. Contr. Zool. 559, 1-85.

Platnick, N. I. (1979). Philosophy and the transformation of cladistics. Syst. Zool. 28, 537-546.

Platnick, N. I. (1993). Character optimization and weighting: differences between the standard and three-taxon approaches to phylogenetic inference. Cladistics 9, 267-272.

Platnick, N. I., Griswold, C. E., and Coddington, J. A. (1991). On missing entries in cladistic analysis. Cladistics 7, 337-343.

Platnick, N. I., Humphries, C. J., Nelson, G., and Williams, D. M. (1996). Is Farris optimization perfect?: three-taxon statements and multiple branching. Cladistics 12, 243-252.

Rieppel, O. C. (1988). "Fundamentals of Comparative Biology". Birkhäuser Verlag, Basel.

Sattath, S., and Tversky, A. (1977). Additive similarity trees. Psychometrika 42, 319-345.

Swofford, D. L. (1993). "PAUP: Phylogenetic Analysis Using Parsimony, Version 3.1.1", Program and Documentation. Illinois Natural History Survey, Champaign.

Swofford, D. L., Olsen, G. J., Wadell, P. J., and Hillis, D. M. (1996). Phylogenetic inference. In "Molecular systematics", 2nd ed. (D. M. Hillis, C. Moritz, and B. K. Mable, Eds), pp. 407-514. Sinauer Associates Inc., Sunderland, Massachusetts.

Swofford, D. L., and Maddison, W. P. (1987). Reconstructing ancestral states under Wagner parsimony. Mathematical Biosciences 87, 199-229.

Udovicic, F., McFadden, G. I., and Ladiges, P. Y. (1995). Phylogeny of Eucalyptus and Angophora based on 5s rDNA spacer sequence data. Mol. Phylogenet. Evol. 4, 247-256.

Wilkinson, M. (1994). Three-taxon statements: when is a parsimony analysis also a clique analysis? Cladistics 10, 221-223. 